\section{Tackling the supreme symmetry}

M. J. Duff

Superspace or One Thousand and One

Lessons in Supersymmetry.

By S.J. Gates Jr, M.T. Grisaru,

M. Roček and W. Siegel.

Benjamin/Cummings: 1983. Pp.548. Hbk

$\$ 48, £ 40.80 ; p b k \$ 25$, £21.25.

HAD Scheherazade tried to while away those Arabian nights with One Thousand and One Lessons in Supersymmetry, one has the feeling that long before the chapter on quantum superspace Schahriar would have cried "Hey, wait a minute! Where are the references?"'. The graduate student of theoretical physics or other inquisitive researcher, hoping to use this text as a guide to the literature on supersymmetry and supergravity, will be sadly disappointed. Not one reference to the original works is to be found in the book. And of the six references to conference proceedings, one is incorrect. (The title of the 1981 Trieste proceedings should be Supergravity 81 and the editors were S. Ferrara and J. G. Taylor.) The authors, all of whom are wellknown Sultans of Supersymmetry, apologize to their friends in the field and beg pardon for omitting the credits. While I suspect that no such pardon will be forthcoming, this is nevertheless a valuable addition to the introductory literature on the ideas of supersymmetry and superspace which will be essential reading for the devotee.

In the words of the Introduction, "Supersymmetry is the supreme symmetry: It unifies spacetime symmetries with internal symmetries, fermions with bosons, and (local supersymmetry) gravity with matter". About one-third of the current publications in theoretical elementary particle physics is devoted to supersymmetry or supergravity, and an increasing number of experimental papers deal with the search for the still unobserved (and perhaps unobservable) superparticles which the theory predicts. More and more $\mathrm{PhD}$ students are choosing this field for their research yet constantly one hears the complaint that there is no adequate starting point for the learner. Superspace will certainly be of help, but it is not for the casual browser or the dilettante.

Supersymmetry experts fall into two categories: those who treat the theory like any other quantum field theory, using separate "component" fields to describe both bosonic (integer spin) and fermionic (odd-half-integer spin) particles; and those who prefer the new-fangled approach in which bosons and fermions are combined into "superfields" which live in a strange world called "superspace". In superspace, the four spacetime dimensions are bosonic with coordinates obeying the usual multiplication $\mathrm{AB}=+\mathrm{BA}$ but extra fermionic dimensions are also introduced whose coordinates obey $\mathrm{AB}=-\mathrm{BA}$.

As their title suggests, the authors of Superspace are unashamedly in favour of these superfields and use the superspace formalism throughout. They have covered all aspects of superspace: superspace geometry, representations of supersymmetry, and global superfields, but their ultimate goal is the unification of gravity and matter via supergravity. At present supergravity is the best available candidate for the Unified Field Theory which will unite gravity with the other fundamental forces of nature. One of the problems in

\section{Jenner by himself}

\section{Donald R. Hopkins}

\section{Letters of Edward Jenner: And Other \\ Documents Concerning the Early \\ History of Vaccination.}

Edited by Genevieve Miller.

Johns Hopkins University Press: 1983.

Pp.145. \$15, £12.75.

THE recent global triumph over smallpox has stimulated great interest in historical aspects of that disease and persons associated with it. So it is the right time finally to have published these letters of Edward Jenner from the Henry Barton Jacobs Collection at Johns Hopkins University. More than anyone else, it was Jenner who set the stage for the eventual eradication of smallpox with his epochal discovery of vaccination in 1796.

Because of Jenner's great historical im- such a scheme is to avoid the ultraviolet divergences of quantum gravity. The authors present a convincing case that only with the techniques of super-Feynman diagrams (for which they themselves have been largely responsible) can we hope to answer this all-important question.

Superspace is an Aladdin's cave of highly-condensed information. But although it is written with an easy style and admirable clarity, it will require a keen mind and determination, rather than a simple "Open, Sesame", to extract the riches.

Michael Duff is a Lecturer in Physics at Imperial College, University of London.

portance and his broad interests, I have often wished I could hear him describe his discovery and the related events that followed, at first-hand, or at least in a taped interview. Fortunately, even though Jenner is of an earlier era and we have no taped interview, we do have many of his letters, some 600 of which are known to still exist. This book contains a good many (110) of them, most being published here for the first time.

As Genevieve Miller notes in her brief introductory essay, in these letters to 45 different correspondents we meet Jenner the naturalist, author, father, country gentleman, scientist, husband, horticulturalist, geologist and the medical practitioner. We also meet a man who, like all of us, was occasionally wildly mistaken. Most interestingly, however, since all but two of these letters are from the period after he discovered vaccination (when he was 47 years old and already had made several significant scientific discoveries

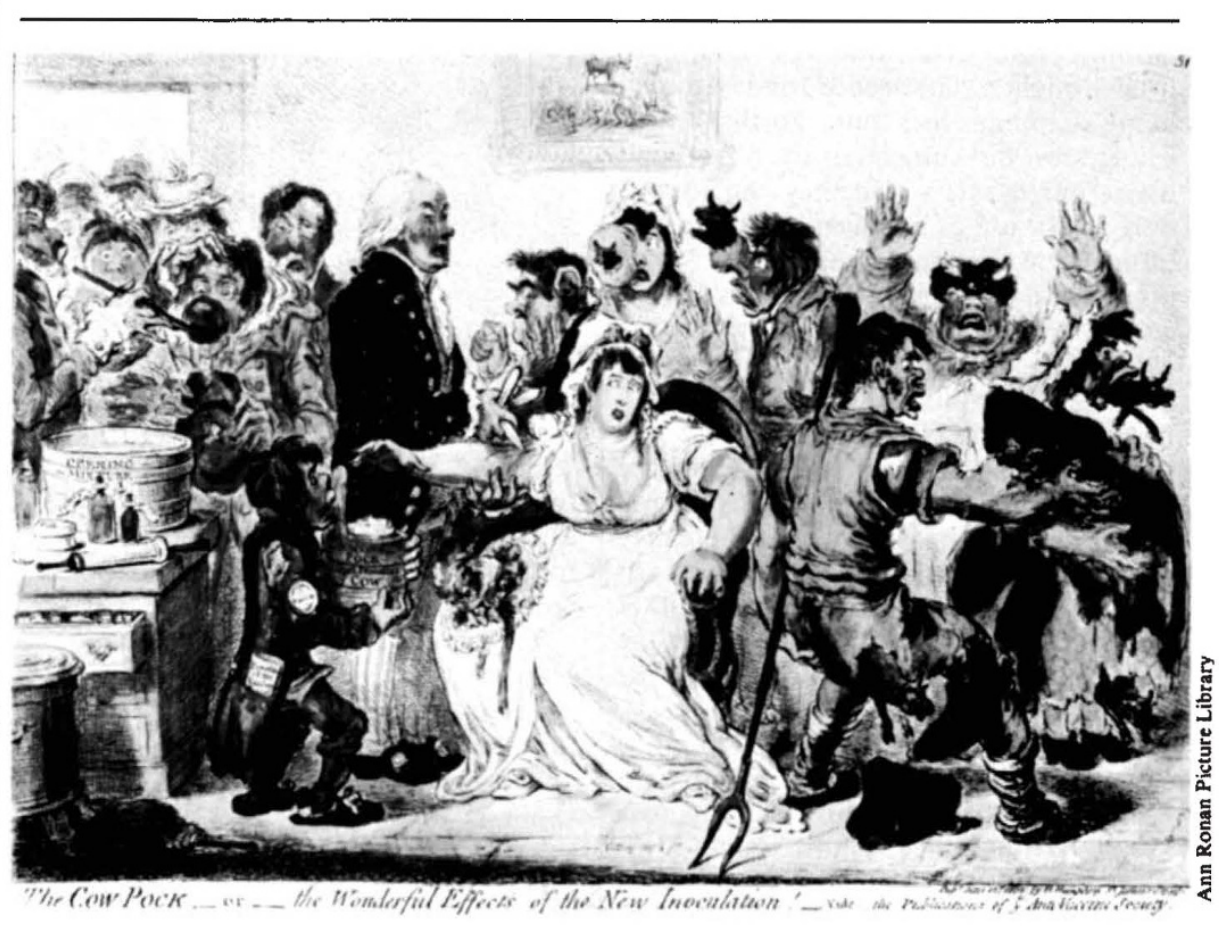

Cow pock and the fears of the public - Gillray's celebrated cartoon of 1802, lampooning the activities of the Anti-Vaccine Society. 